\title{
On the Transient Response and the Frequency Analysis of Transmission Line Towers
}

\author{
Mohamed M. Saied \\ Electrical Engineering Department, College of Engineering and Petroleum, Kuwait University, \\ P.O. Box 5959, Code 13060, Kuwait City, Kuwait
}

Correspondence should be addressed to Mohamed M. Saied; mmsaied2@yahoo.com

Received 26 February 2013; Accepted 24 March 2013

Academic Editors: A. Cardou and J. J. González de la Rosa

Copyright (C) 2013 Mohamed M. Saied. This is an open access article distributed under the Creative Commons Attribution License, which permits unrestricted use, distribution, and reproduction in any medium, provided the original work is properly cited.

\begin{abstract}
This paper proposes an approach to the study of the transient response and the frequency characteristics of power lines' towers, when subjected to lightening strikes. It starts with dividing the nonuniform line representing the tower into a number of sections. From the usually known dependence of the tower's characteristic impedance on the vertical coordinate and the application of a recursive circuit reduction technique, an s-domain expression for the tower input impedance can be obtained. This expression, followed by the numerical Laplace inversion, is utilized for the determination of the tower's transient response. The impedance expression can be also used to determine the tower's resonance frequencies. This was used to demonstrate some potentially critical situations in which the tower is hit by lightning strikes comprising multiple current pulses. The validity of the proposed technique is demonstrated by comparing the achieved results with those already available in the literature for the same case studies.
\end{abstract}

\section{Introduction}

The proper insulation coordination and overvoltage protection of power networks and components will depend on their topology and their characterizing circuit parameters as well as on the time waveform of the input voltage and/or current stimuli. The accurate determination of the expected transient stresses will allow more effective protective measures and more efficient utilization of the network components. In the recent literature, considerable attention was paid to the transient phenomena involving overhead lines and underground cables [1-13]. Although most of these investigations are dealing with the active (live) line conductors, the ground wires, and the insulating equipment, many interesting studies dealing with the line towers and their steel structures were also conducted [6-13]. An important issue in this regard is the transient behaviour of the transmission lines' towers after being hit by lightening strikes

In terms of circuit analysis, the towers are treated as nonuniform transmission lines with location-dependent inductance and capacitances per unit length. There are three well-established techniques for the analyzing tower transients.
(1) The time domain simulation based on dividing the tower into a number of sections represented by Lequivalent circuits. A set of simultaneous differential equations in the state variables (the inductor currents and capacitor voltages) are formulated and then solved numerically $[1,3]$. This method allows the consideration of losses and nonlinear phenomena such as corona $[4,5,13]$.

(2) Travelling wave technique, extending the concepts of the reflection and refraction coefficients (which will then be location dependent along the tower) as well as the Bewley lattice diagram $[1,12]$. This approach is best suited for cases in which the transmission elements can be assumed lossless and in which both the sources and the termination impedances can be assumed pure resistive. This leads to real reflection and refraction coefficients.

(3) The Laplace or s-domain approach, example of which is first documented in reference [6] and then in [11]. Starting with the application of curve fitting methods, a suitable (usually exponential) function approximating the location dependence of the tower 
characteristic (or surge) impedance could be derived. Closed-form s-domain expressions for the currents and voltages at any point of interest along the tower could then be found analytically. These s-domain expressions, together with the numerical inverse Laplace transform, were utilized for the determination of the tower's time-domain transient response, for any tower footing impedance, any lightening voltage or current time waveform, and any source impedance.

A main limitation affecting the application of the sdomain approach to the study of tower transients is the inevitable error introduced by the curve fitting of the characteristic impedance expressed as a function of location. In this paper, a technique will be presented allowing the sdomain and, hence, the time-domain analyses of the tower to be conducted without resorting to any approximation for the location dependency of its characteristic impedance. It suggests a recursive circuit reduction procedure capable of providing a closed-form expression for the tower input impedance, for any internal impedance of the lightening discharge. This source impedance as well as that of the tower footing, can assume any complex values. The next step is to apply the numerical inverse Laplace transform in order to get the time waveforms of the voltage and current at the tower's top in response to typical single- or multipulse lightening strikes. Furthermore, the above expression of the tower input impedance will be used for identifying the tower's resonance and antiresonance frequencies.

\section{Method of Analysis}

Figure 1 illustrates the tower under investigation. As indicated to the right, its height is $H$ meters and its general footing impedance is denoted $Z_{o}$. It is hit by a direct lightening strike at its top. It is required to find the time waveform of the voltage $v_{T}(t)$ and current $i_{T}(t)$ at the tower's top as the wave propagates along the tower. The lightening strike is represented by its Norton's equivalent circuit to the left, comprising a current source $i_{s}(t)$ of known magnitude and waveform and a parallel connected internal resistance $R_{i}$. Nevertheless, the approach is also valid for any complex value for the internal impedance. Due to wave nature and the long path of the lightening strike, the current $i_{s}(t)$, applied at $t=0$, should be double the injected lightening current. Physically, the inductance per unit $L$ at any point along the tower increases with the coordinate $x$. On the other hand, the capacitance per unit length, $C$, behaves exactly the opposite. Accordingly, the tower's characteristic (or surge) impedance $Z_{c}=\sqrt{L / C}$ will increase with the coordinate $x$. The following relation between $Z_{c}$ and $x$ is adopted from $[6,12]$ :

$$
Z_{c}=50+35 \sqrt{x}
$$

It increases from $50 \Omega$ at the ground level to $353.1 \Omega$ at the top of the tower of the height $H=75 \mathrm{~m}$. The propagation time along the tower is accordingly $T=0.25 \mu \mathrm{s}$.

The analysis starts with dividing the nonuniform line representing the tower in $N$ sections of $d=H / N$ meters length. Increasing the number of sections $N$ will enhance the accuracy. Theoretically, there is no upper bound for $N$. It is limited only by the available computational resources. A value $N=25$ sections was assumed in this study. Accordingly, each section is of $3 \mathrm{~m}$ length. Each of the section's two ports will be identified by an integer coordinate $n$. Figure 2 depicts the tower section between the $n$th and the $(n+1)$ th ports.

By inspecting Figure 2 and after some circuit manipulations, the values of the two input impedances $Z_{\text {input, }(n)}(s)$ and $Z_{\text {input, }(n+1)}(s)$ as seen at the lower and upper terminal pairs $n$ and $(n+1)$, respectively, are related by the recursive equation:

$$
Z_{\text {input },(n+1)}(s)=\frac{s k Z_{c} d+Z_{\text {input }, n}(s)}{1+(s k d)^{2}+d s\left(k / Z_{c}\right) Z_{\text {input }, n}(s)},
$$

where $k=10^{-8} / 3 \mathrm{sec} / \mathrm{m}, d=H / N \mathrm{~m}$, and, as a result of (1)

$$
Z_{c}=50+35 \sqrt{\frac{75 n}{N}}
$$

Equation (2) takes into account that the inductance $L$ and capacitance $C$ per meter can be expressed as $C=k / Z_{c}$ and $=k Z_{c}$, respectively.

Starting from the ground level $(n=0)$ and with the initial value $Z(0)=$ the known tower footing impedance $Z_{o}$, (3) can be applied repeatedly in order to get the values of $Z_{\text {input, } 1}(s), Z_{\text {input, } 2}(s), Z_{\text {input,3}}(s), \ldots$ up to the required input impedance at the tower top $Z_{T}(s)=Z_{\text {input, } N}(s)$ as a function of the tower data and the complex frequencys. The frequency response of the input impedance can then be found by substituting $s=j \omega$.

Applying the current divider rule at the tower top, the following equation can be derived:

$$
\begin{gathered}
V_{T}(s)=I_{s}(s) \frac{R_{i}}{\left[R_{i} / Z_{T}(s)\right]+1}, \\
I_{T}(s)=\frac{I_{s}(s)}{\left[Z_{T}(s) / R_{i}\right]+1},
\end{gathered}
$$

where $I_{s}(s), V_{T}(s), I_{T}(s)$ are the Laplace transforms of the current source in the lightening's Norton equivalent circuit, the voltage at the tower top, and the current injected at that top, respectively. The resistance $R_{i}$ represents the source shunt resistance of the lightning's equivalent circuit.

Using one of the efficient algorithms for performing the numerical inverse Laplace transform, the time response of the voltage and current at the tower top, that is, $v_{T}(t)$ and $i_{T}(t)$, respectively, can be obtained. More details on the Hosono algorithm can be found in $[6,7]$.

\section{Sample Results}

The validity of the proposed technique is verified by comparing the achieved results with those already available in the literature for the same case studies.

The results of the first test case are illustrated in Figure 3. It deals with applying a step-shaped $30 \mathrm{kA}$ lightening current at $t=0.1 \mu \mathrm{s}$. The assumed number of the tower sections 


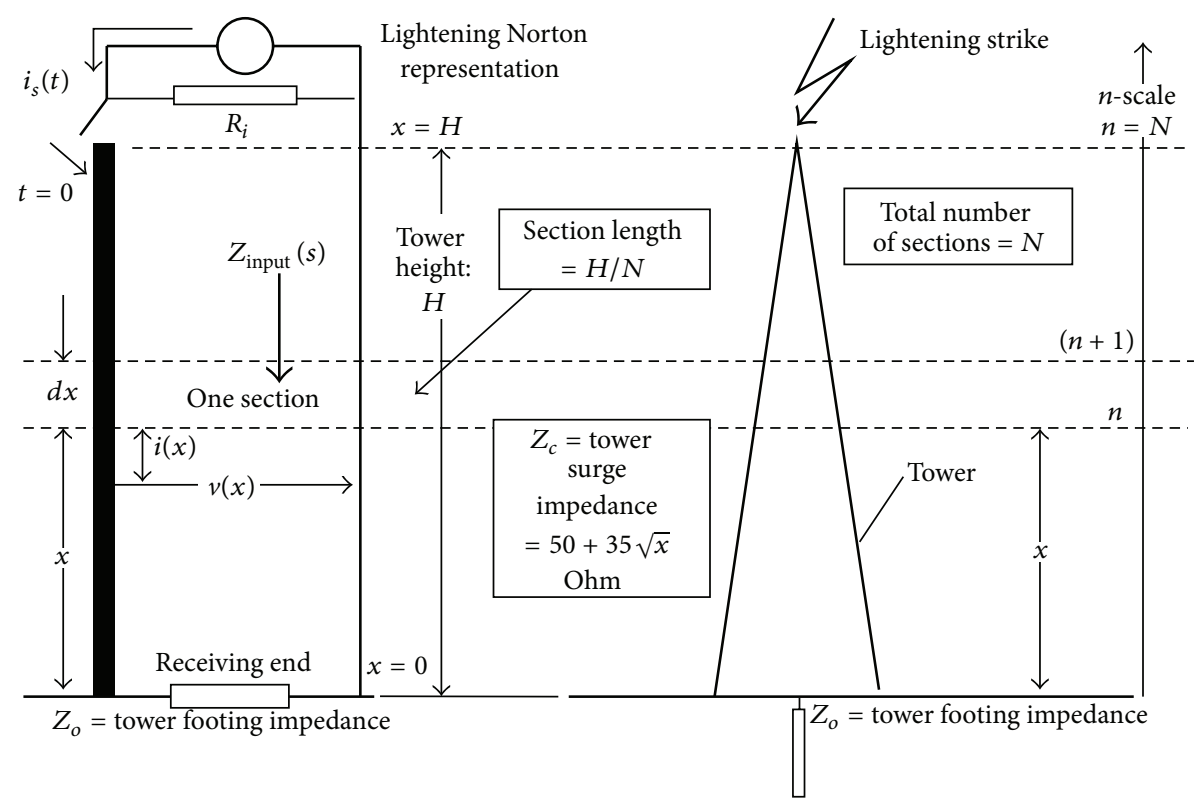

FIgURE 1: The tower under study and its circuit representation.

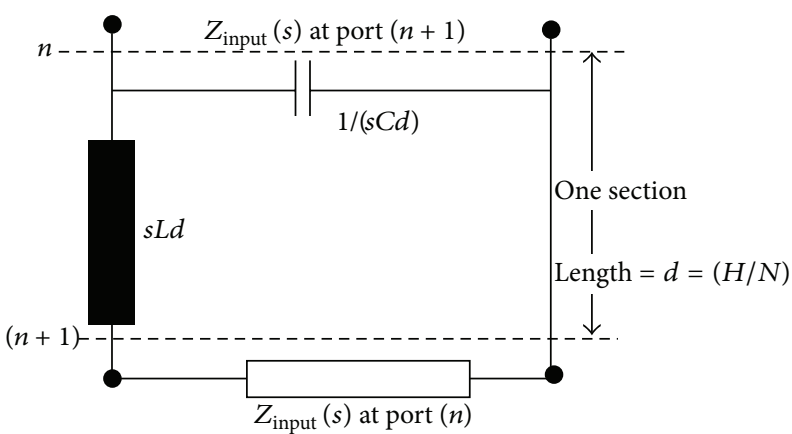

FIGURE 2: The s-domain equivalent circuit of the tower section between the two ports $n$ and $(n+1)$.

is $N=25$. The source resistance is $R_{i}=500 \Omega$. The tower footing resistance is $Z_{o}=10 \Omega$ pure resistive. For this special case, the line is assumed uniform with the constant characteristic impedance $=250 \Omega$. Immediately after $t=$ $0.1 \mu \mathrm{s}$, the voltage and current at the tower top, that is, $v_{T}(t), i_{T}(t)$, jump to exactly $10 \mathrm{MV}$ and $400 \mathrm{~A}$, respectively. These values can be verified using simple DC circuit analysis. The rapid voltage decrease and current increase after elapsing double the tower delay time, that is, immediately after $t=$ $0.6 \mu \mathrm{s}$, can be clearly recognized. This is attributed to the negative voltage reflection coefficient when the first wave reaches $x=0$, since the tower footing resistance $\left(Z_{o}=\right.$ $10 \Omega$ ) is less than the tower surge impedance at that point $\left(Z_{c}=250 \Omega\right)$. At $t=\infty$ and from simple DC analysis, the voltage and current at the tower top $v_{T}(t), i_{T}(t)$ should assume the values $0.588 \mathrm{MV}$ and $58.8 \mathrm{kA}$, respectively. These values agree with the final values shown by the two plots of Figure 4. It should be noted that the same results could have been obtained through the application of the Bewley lattice diagram.

The next section deals with a second test case. Reference [6] presents the results of applying the time-domain analysis after dividing the tower into $N=7$ sections. It has the nonuniform surge impedance distribution according to (1). The lightening internal resistance $R_{i}$ and the tower footing resistance are assumed to be $250 \Omega$ and $10 \Omega$ (pure real), respectively. The lightening surge current $i_{s}(t)$ is of a peak value of $30 \mathrm{kA}$ and follows the double exponential equation:

$$
i_{s}(t)=30397\left[e^{-10^{6} t / 17.63}-e^{-10^{6} t / 0.0316}\right] .
$$

Its Laplace transform is

$$
I_{s}(s)=30397\left[\frac{1}{(56721.5+s)}-\frac{1}{(31645600+s)}\right] .
$$

The results in [6] are in good agreement with the plots shown in Figure 4 below for the same case but obtained by applying the here-suggested recursive circuit reduction technique. Each plot reflects the computation of 10000 points (or numerical inverse Laplace transforms) over the time span of $1 \mu \mathrm{s}$. The maximum values of the voltage and current $v_{T}(t), i_{T}(t)$ at the tower top are about 8.5 MV and $61 \mathrm{kA}$, respectively. The rapid voltage decrease and current increase after elapsing double the tower delay time, that is, after $t=$ $0.5 \mu \mathrm{s}$, can be clearly recognized. This is attributed to the negative voltage reflection coefficient when the first wave reaches $x=0$.

As mentioned earlier, the accuracy of the suggested procedure increases with the assumed number of the tower sections $N$. To demonstrate this fact, the case of $30 \mathrm{kA}$ double exponential surge current hitting the top of the nonuniform tower (leading to the results depicted in Figure 4 using $N=7$ 


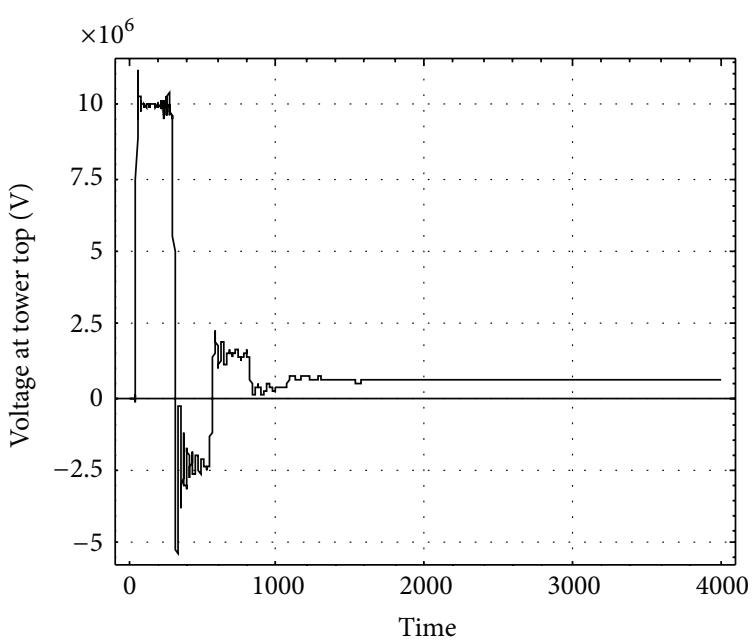

(a)

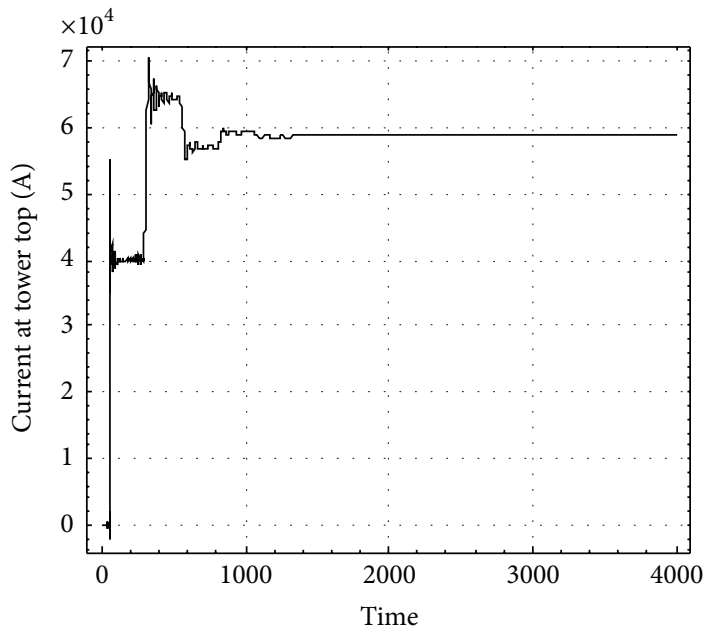

(b)

FIgure 3: The voltage and current at the tower top $v_{T}(t), i_{T}(t)$ resulting from applying the suggested approach due to a $30 \mathrm{kA}$ step lightening current surge applied at $t=0.1 \mu \mathrm{s}$. Number of sections $N=25$ (4000 divisions $=8 \mu \mathrm{s})$.

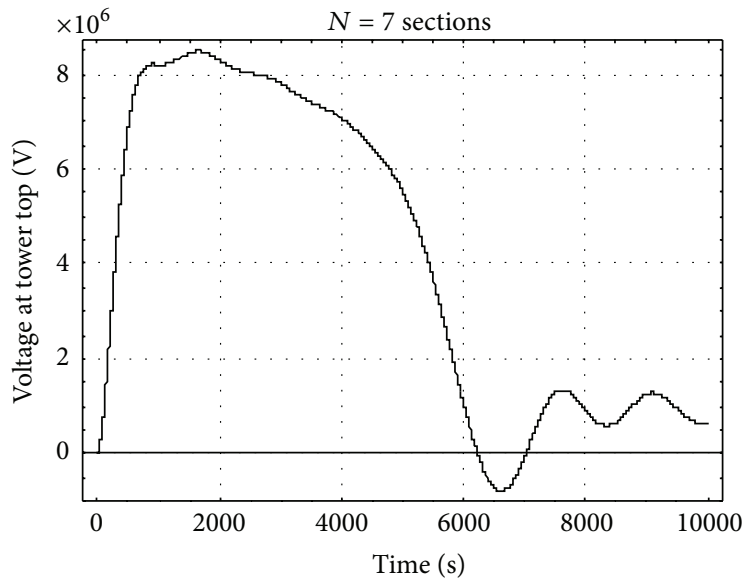

(a)

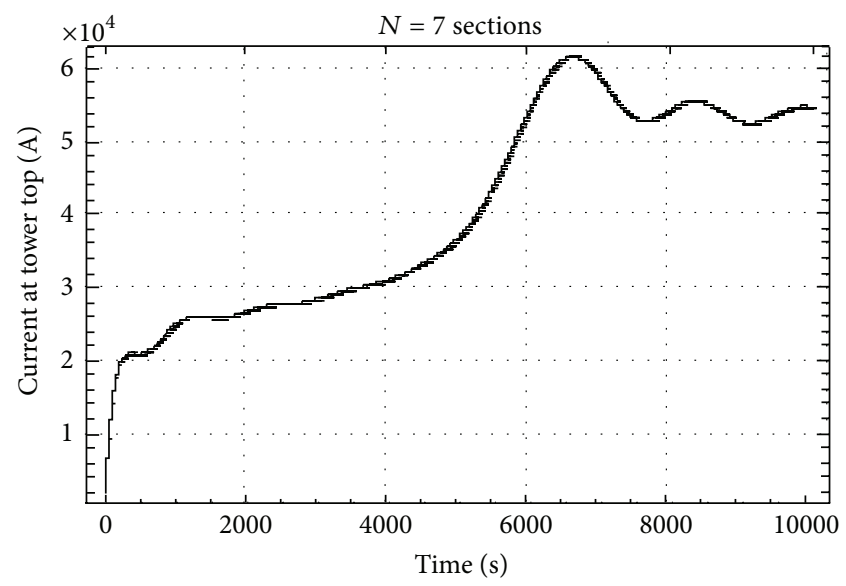

(b)

Figure 4: The voltage and current at the tower top $v_{T}(t), i_{T}(t)$ resulting from applying the suggested approach due to a $30 \mathrm{kA}$ double exponential lightening current surge. Number of sections $N=7$ (10000 divisions $=1 \mu \mathrm{s})$.

sections) will now be repeated but with the much larger value of $N=25$ sections. The results for both values of the number of sections $N$ are shown in Figure 5 over the same time range of $6 \mu \mathrm{s}$. In addition to the voltage and current at the tower top, $v_{T}(t), i_{T}(t)$, the commonly used term of the instantaneous input impedance defined as $z_{T}(t)=\left|v_{T}(t) / i_{T}(t)\right|$ is also shown. Each plot reflects the computation of 30000 points (or numerical inverse Laplace transforms) over the time span of $6 \mu \mathrm{s}$. It is seen that the use of the larger number of sections $N=25$ leads to a more accurate and faithful representation of the reflections felt at the tower top after $2 T, 4 T, 6 T$, where $T$ is the tower delay time $=0.25 \mu \mathrm{s}$.

The results of applying the proposed recursive circuit reduction with $N=25$ are much closer to the results given in [12] for the travelling wave solution if compared with the plots documented in [6] for the s-domain solution after approximating (1) by an exponential function.

Figure 6 illustrates the impulse response of the voltage at the tower top $v_{T}(t)$. The current source representing the lightening discharge is expressed by an impulse or Dirac function $i_{s}(t)=\delta(t)$ of infinite internal resistance $R_{i}=\infty$. It follows that in the s-domain $I_{s}(s)=1$, and that the voltage at the tower top, $v_{T}(t)$, is the inverse Laplace transform of $Z_{T}(s)$ determined earlier for the assumed tower-footing resistance of $10 \Omega$. As expected, the computation results for this special case indicate that the current at the tower top $i_{T}(t)$ is zero for $t>0$. The reflections felt at the tower top after $2 T, 4 T, 6 T$, and so forth, where $T$ is the tower delay time $(0.25 \mu \mathrm{s})$, are clearly recognized. The voltage plot demonstrates the tower's natural response and exhibits several natural frequencies. By 

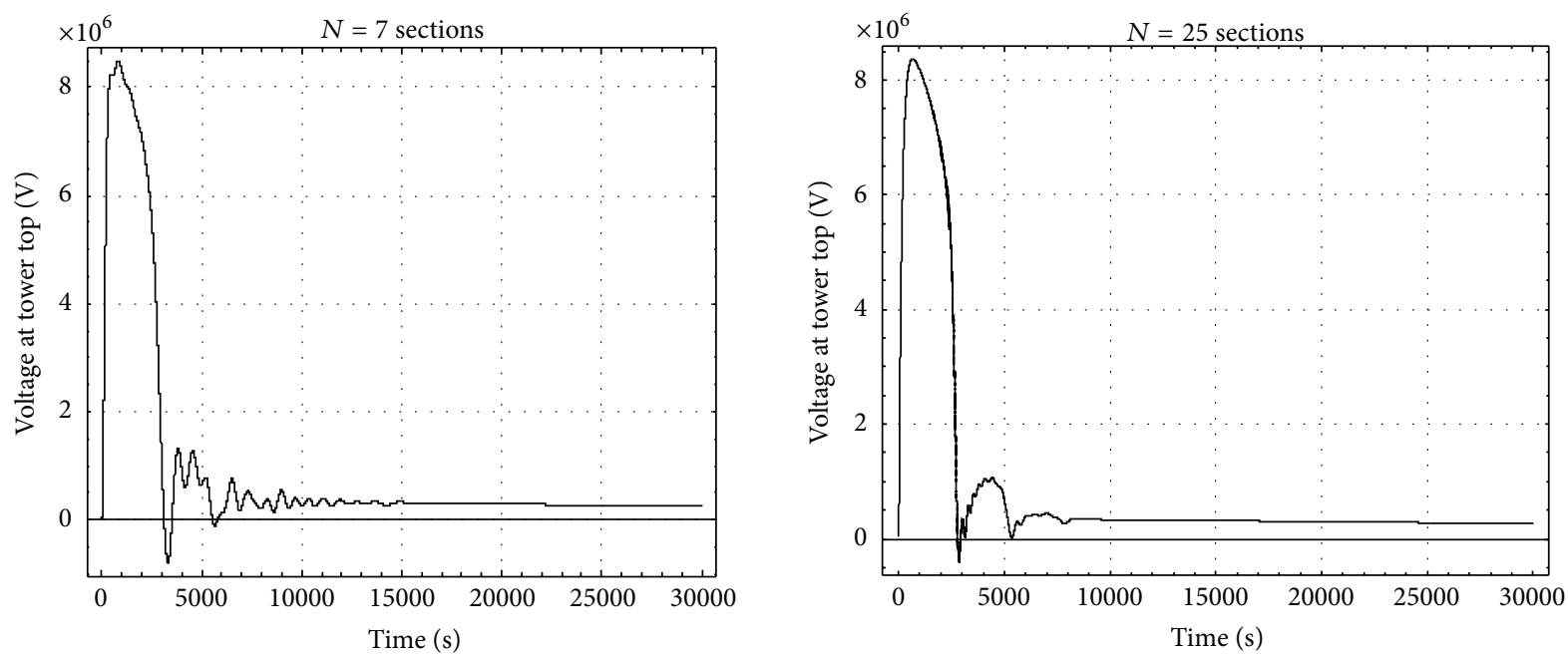

(a) Voltage at the tower top
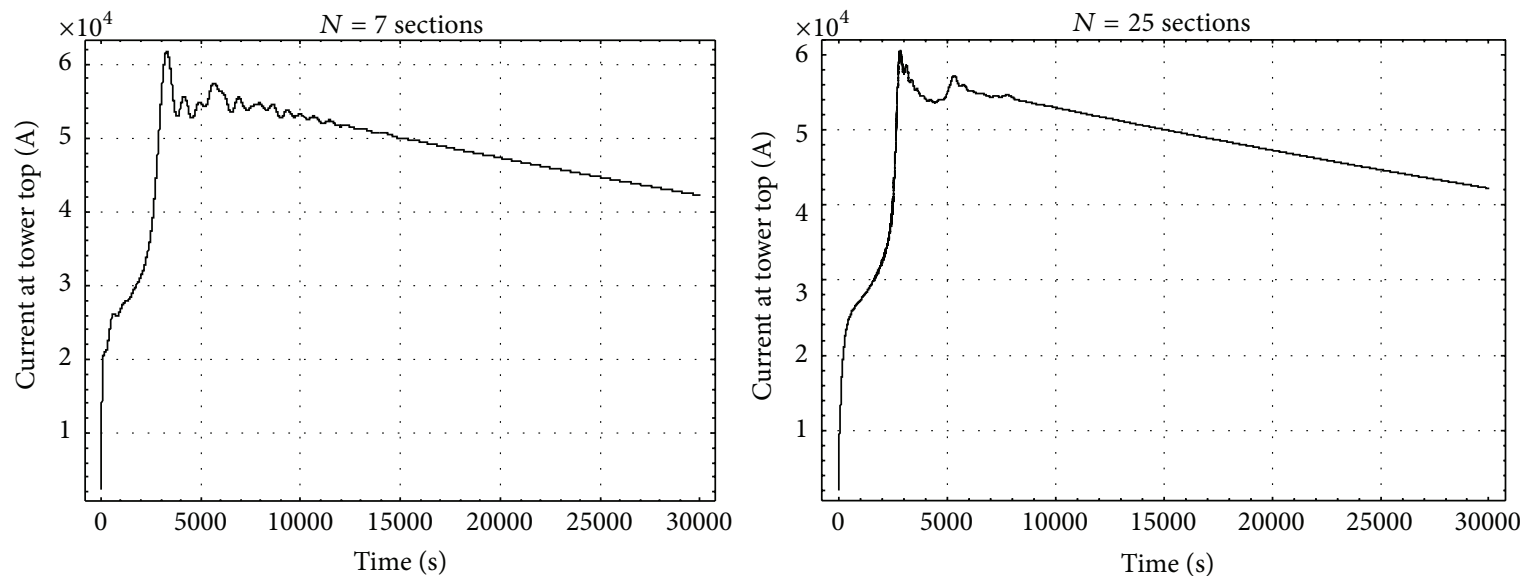

(b) Current at the tower top
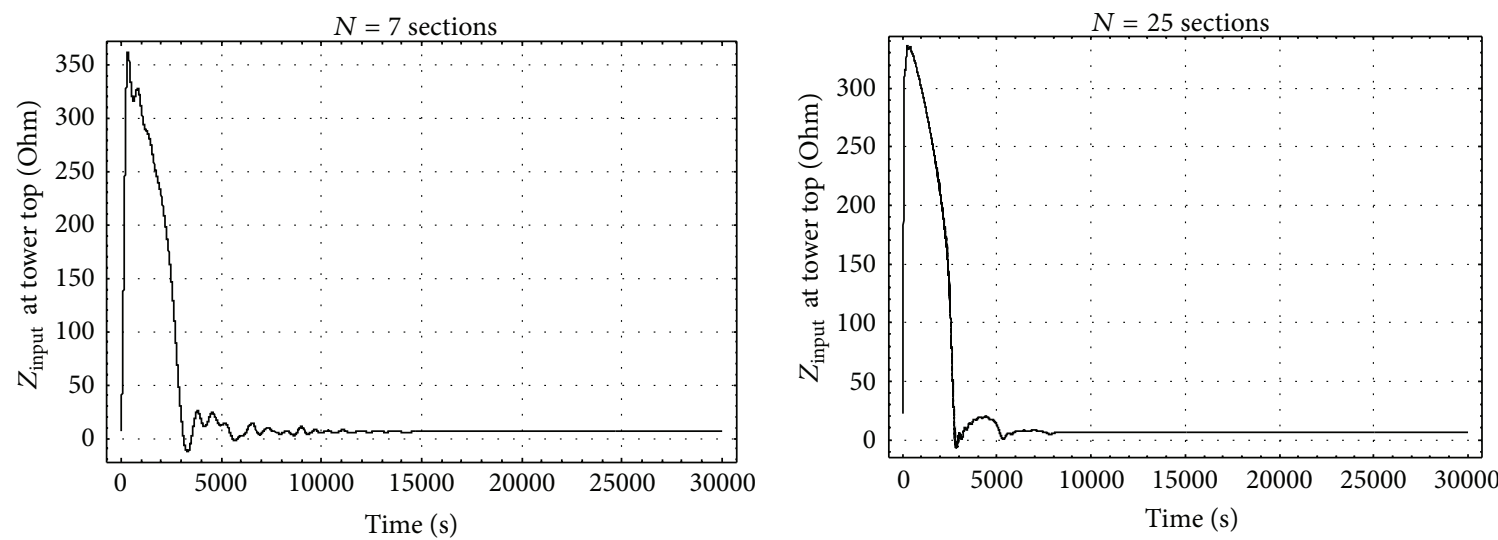

(c) Instantaneous input impedance seen at the tower top

FIGURE 5: A comparison between the results of the voltage and the current at the tower top as well as the instantaneous tower input impedance resulting from applying the suggested approach due to a $30 \mathrm{kA}$ double exponential lightening current surge. Numbers of sections are $N=7$ for the plots to the left and $N=25$ for the plots to the right ( 30000 divisions $=6 \mu \mathrm{s}$ ). 


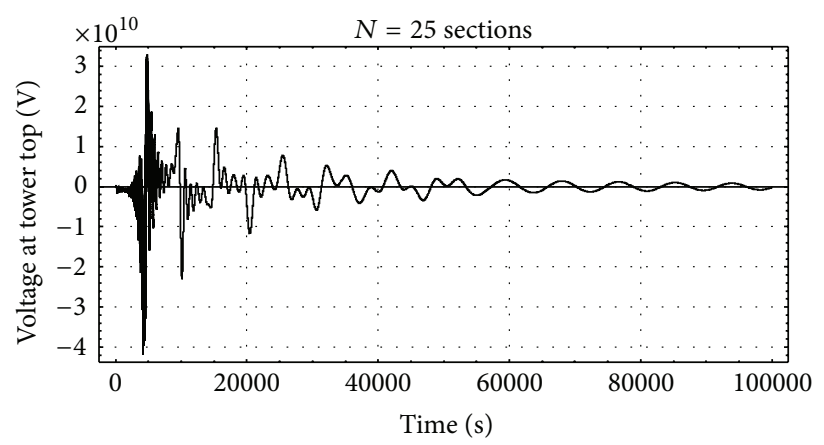

FiguRE 6: The voltage at the tower top $v_{T}(t)$ resulting from applying the suggested approach due to a lightening current surge of one Dirac function, that is, $i_{s}(t)=\delta(t)$. The source internal resistance $R_{i}$ was assumed infinity. Number of sections $N=25$ (100000 divisions $\left.=10 \mu \mathrm{s}\right)$.

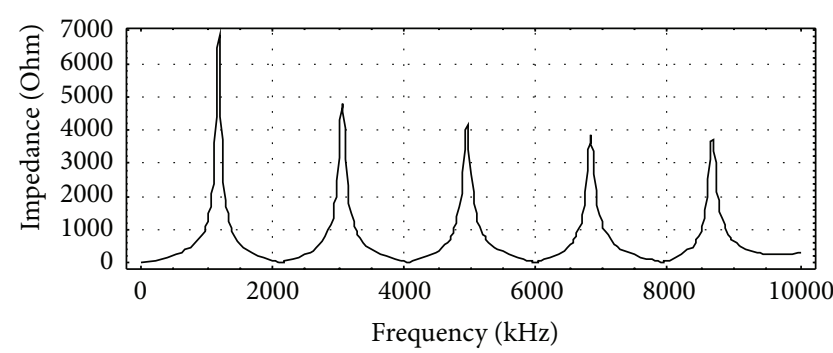

(a) Impedance magnitude

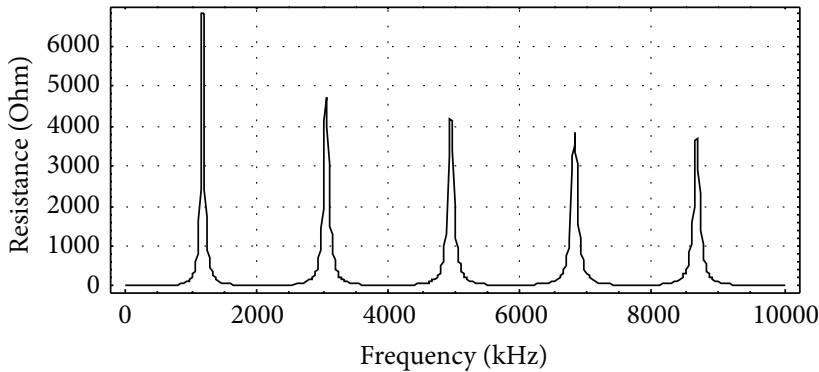

(b) Real part

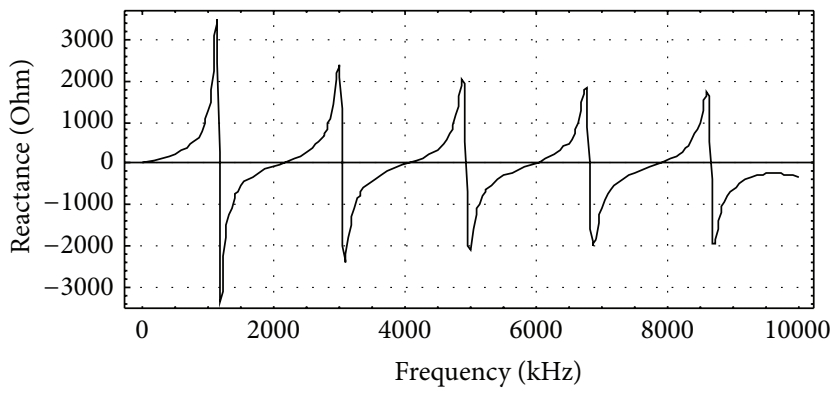

(c) Imaginary part

FIGURE 7: The frequency response of the input impedance $Z_{T}(j \omega)$ over the frequency range $0-10000 \mathrm{kHz}$.

inspection, the lowest natural frequency prevailing for $t \geq$ $5 \mu \mathrm{s}$ is about $1200 \mathrm{kHz}$.

The next section deals with the frequency response of the input impedance at the tower top $Z_{T}(s)=Z_{\text {input }, N}(s)$ which can be found by substituting $s=j \omega$.

The three plots (a), (b) and (c) in Figure 7 depict the frequency dependence of the impedance magnitude, its real and imaginary parts. The maxima and minima of the impedance magnitude, as recognized in Figure 7(a), represent parallel and series resonances, respectively. The corresponding values of the resonance frequencies can be determined from the sign reversals of the impedance phase angle, as Figure 7(c) indicates.

It is observed that the tower exhibits 9 resonance frequencies, five of them are parallel ones associated with impedance maxima (several $\mathrm{k} \Omega$ ). The remaining four are series resonance frequencies exhibiting minimum impedance magnitude (several $\Omega$ ). No resonance frequencies are detected above $8000 \mathrm{kHz}$. At all resonance frequencies, the input impedances at the tower top are purely resistive. The results are summarized in Table 1.

The two upper plots in Figure 8(a) illustrate the voltage and current $v_{T}(t), i_{T}(t)$ at the tower top due to a multipulse lightening discharge represented by a current source including seven equidistant pulses of magnitude $1 \mathrm{~A}$ and internal impedance $250 \Omega$. The tower-footing resistance is assumed $10 \Omega$. The number of sections is $N=25$. The pulse separation is taken to be as $0.8547 \mu$ s leading to the pulse frequency $1170 \mathrm{kHz}$, coinciding with the first parallel resonance frequency in Table 1. From the table, the tower input impedance at this frequency is $6900 \Omega$ (for sinusoidal excitation). The results show relatively high voltage values (around $270 \mathrm{~V}$ ) and low current values (around 0.9 A) during the first $6.5 \mu \mathrm{s}$. 

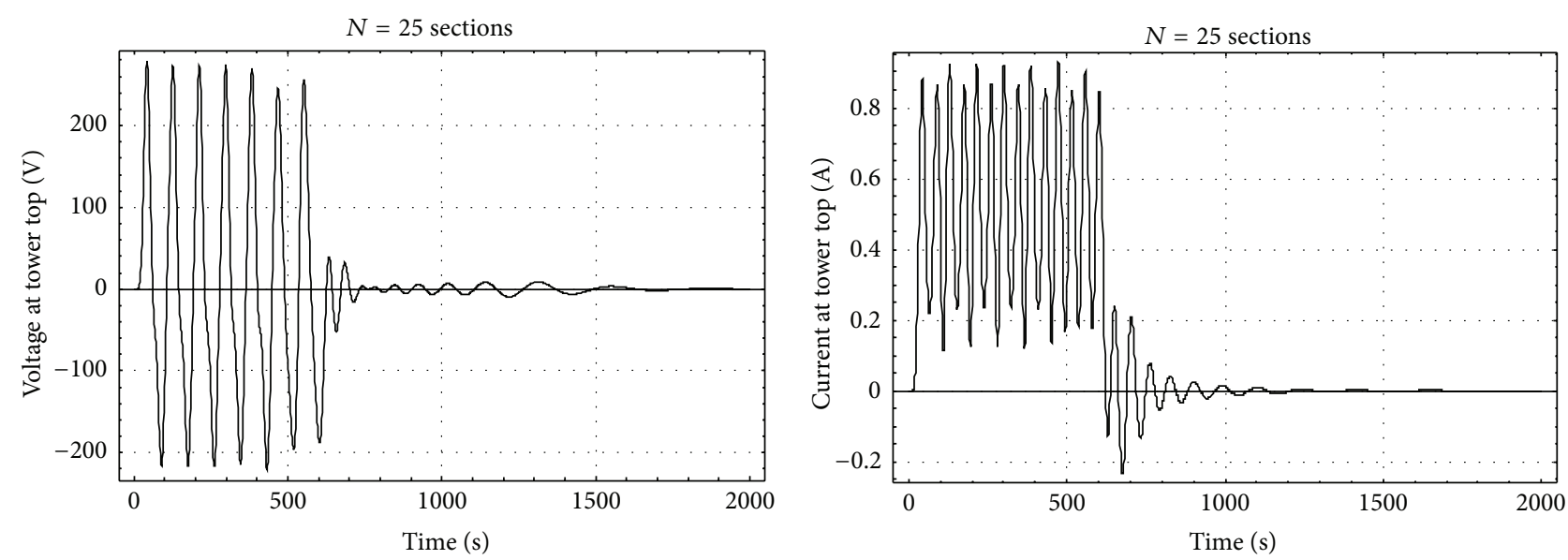

(a) Tower response to 7 equidistant lightening pulses with pulse rate corresponding to the first parallel resonance frequency $1170 \mathrm{kHz}$ (time scale: 2000 divisions $=20 \mu \mathrm{s})$
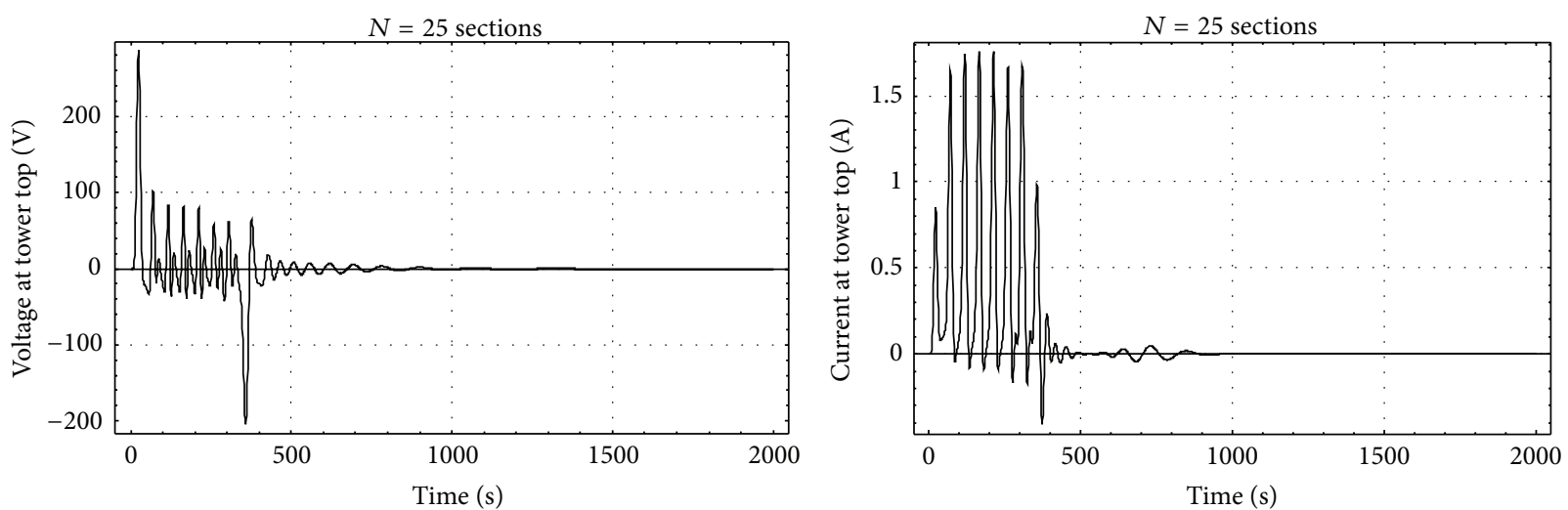

(b) Tower response to 7 equidistant lightening pulses with pulse rate corresponding to the first series resonance frequency $2110.2 \mathrm{kHz}$ (time scale: 2000 divisions $=20 \mu \mathrm{s})$

FiguRE 8: Tower response to 7 equidistant lightening pulses with pulse rate corresponding to the first parallel resonance frequency $1170 \mathrm{kHz}$ ((a) upper plots) and the first series resonance frequency $2110.2 \mathrm{kHz}$ ((b) lower plots).

The two lower plots in Figure 8(b) show the voltage and current $v_{T}(t), i_{T}(t)$ at the tower top due to a multipulse lightening discharge represented by a current source including seven equidistant pulses of magnitude $1 \mathrm{~A}$ and internal impedance $250 \Omega$. The pulse separation here is taken as $0.474 \mu$ s leading to the pulse frequency $2110.2 \mathrm{kHz}$, coinciding with the first series resonance frequency in Table 1 , at which the tower input impedance is $23.53 \Omega$. The results show relatively lower voltage magnitudes and high current values (around $1.8 \mathrm{~A}$ ) during the first $6.5 \mu \mathrm{s}$.

\section{Conclusions}

(1) A new approach to the study of the transient response and the frequency characteristics of the overhead line towers, when subjected to lightening strikes, is presented. The towers are treated as nonuniform lines with location-dependent inductance and capacitances per unit length.

(2) Through the application of a recursive circuit reduction technique, a closed form s-domain expression for the tower input impedance can be derived. This expression, in connection with the numerical inverse Laplace transform, is utilized for determining the tower's time domain response. It can be also used to determine the tower's series and parallel resonance frequencies and to demonstrate some potentially critical situations if the tower is hit by lightning strikes comprising multiple current pulses.

(3) The presented technique allows the s-domain and time-domain analyses of the tower to be conducted without resorting to any approximation in the location dependency of its characteristic impedance. Moreover, the source impedance representing the lightening discharge as well as that of the tower footing can assume any complex values.

(4) The validity of the proposed technique is demonstrated by comparing the achieved results with those already available in the literature for the same case studies. The accuracy of the suggested procedure increases with the assumed number of the tower 
TABLE 1: The resonance frequencies of the tower input impedance $Z_{T}(j \omega)$ and the corresponding impedance values.

\begin{tabular}{|c|c|c|c|c|}
\hline Serial number & $\begin{array}{l}\text { Parallel resonance } \\
\text { frequency } \mathrm{kHz}\end{array}$ & $\begin{array}{l}\text { Parallel resonance } \\
\text { impedance Ohm }\end{array}$ & $\begin{array}{l}\text { Series resonance } \\
\text { frequency } \mathrm{kHz}\end{array}$ & $\begin{array}{l}\text { Series resonance } \\
\text { impedance } \mathrm{Ohm}\end{array}$ \\
\hline 1 & 1170 & 6900 & & \\
\hline 2 & & & 2110.2 & 23.53 \\
\hline 3 & 3051.7 & 4820 & & \\
\hline 4 & & & 4056.8 & 28.89 \\
\hline 5 & 4942 & 4150 & & \\
\hline 6 & & & 6000 & 32.50 \\
\hline 7 & 6825 & 3850 & & \\
\hline 8 & & & 7897 & 34.2 \\
\hline 9 & 8672 & 3740 & & \\
\hline
\end{tabular}

sections. The use of the larger number of sections leads to a more accurate and faithful representation of the voltage and current reflections felt at the tower.

(5) If the current source representing the lightening discharge is expressed by a Dirac function, the voltage waveform at the tower top will be the inverse Laplace transform of the tower input impedance. It demonstrates the tower's natural response and exhibits several natural frequencies.

(6) The analyzed tower exhibits 9 resonance frequencies; five of them are parallel ones associated with impedance maxima (several $\mathrm{k} \Omega$ ). The remaining four are series resonance frequencies exhibiting minimum impedance magnitudes (several $\Omega$ ). No resonance frequencies are detected above $8000 \mathrm{kHz}$

(7) The voltage and current at the tower top due to a multipulse lightening discharge are investigated. The pulse separation is assumed to be $0.8547 \mu \mathrm{s}$ corresponding to the pulse frequency $1170 \mathrm{kHz}$ (the first parallel resonance). The results show relatively high voltage values and low current values during the first $6.5 \mu \mathrm{s}$. The same signals were studied with a pulse separation of $0.474 \mu$ s leading to the frequency $2110.2 \mathrm{kHz}$ (the first series resonance), at which the tower input impedance at this frequency is $23.53 \Omega$. The results show relatively lower voltage magnitudes and high current values.

\section{References}

[1] A. Greenwood, Electrical Transients in Power Systems, chapters 9, 14, Wiley-Interscience, 2nd edition, 1991.

[2] L. Souza, A. Lima, and S. Carneiro Jr., "Modeling overhead transmission line with large asymmetrical spans," in Proceedings of the International Conference on Power System Transients (IPST '11), Delft, the Netherlands, June 2011.

[3] A. I. R. Vazquez, Advanced models for electromagnetic transient simulation of power transmission lines [Ph.D. thesis], University of Guadalajara, Guadalajara, Mexico, 2001.

[4] M. M. Saied and Y. A. Safar, "Electromagnetic transients on compensated lines under corona," Electric Machines and Power Systems, vol. 16, no. 6, pp. 441-462, 1989.
[5] H. Nafisi, M. Davari, and B. Vahidi, "Corona and electromagnetic transient study in overhead transmission lines," in Proceedings of the 2nd International Conference on Modern Power Systems (MPS '08), pp. 174-178, Cluj-Napoca, Romania, November 2008.

[6] M. M. Saied, A. S. AlFuhaid, and M. E. El-Shandwily, "s-domain analysis of electromagnetic transients on nonuniform lines," IEEE Transactions on Power Delivery, vol. 5, no. 4, pp. 20722083, 1990.

[7] M. M. Saied, "Electromagnetic transients on power lines due to multiple-pulse lightening surges," in Proceedings of the CIGRE Session, Paper no. 33-101, Paris, France, 2002.

[8] M. S. Mamis and M. Koskal, "Computation of lightning overvoltages using nonuniform, single-phase line model," in Proceedings of the International Conference on Power System Transients (IPST '99), pp. 406-409, Budapest, Hungary, June 1999.

[9] L. Grcev and F. Rachidi, "On tower impedances for transient analysis," IEEE Transactions on Power Delivery, vol. 19, no. 3, pp. 1238-1244, 2004.

[10] A. Soares, M. A. O. Schroeder, and S. Visacro, "Transient voltages in transmission lines caused by direct lightning strikes," IEEE Transactions on Power Delivery, vol. 20, no. 2, pp. 14471452, 2005.

[11] A. Kaygusuz, S. Mamis, and E. Akin, "s-domain analysis of lightening surge response of a transmission tower with phase conductors," in Proceedings of the International Conference on Power Systems Transients (IPST '03), New Orleans, La, USA, 2003.

[12] C. Menemenlis and Z. T. Chun, "Wave propagation on non uniform lines," IEEE Transactions on Power Apparatus and Systems, vol. 101, no. 4, pp. 833-839, 1982.

[13] M. S. Mamis, "State-space transient analysis of single-phase transmission lines with corona," in Proceedings of the International Conference on Power Systems Transients (IPST '03), New Orleans, La, USA, 2003. 

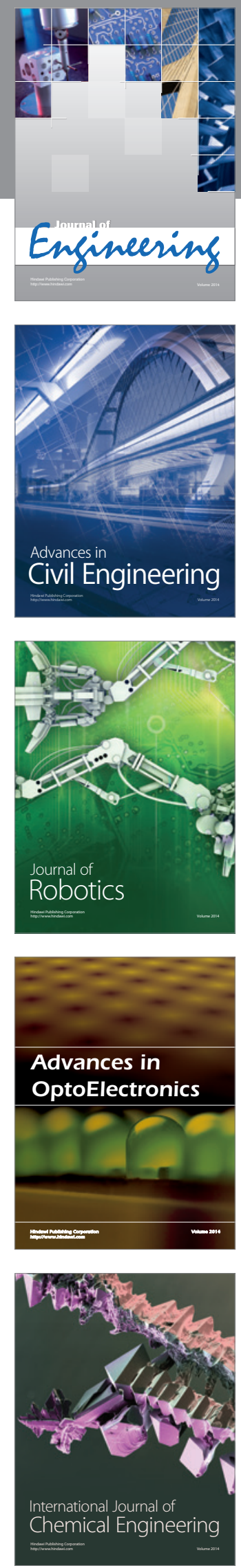

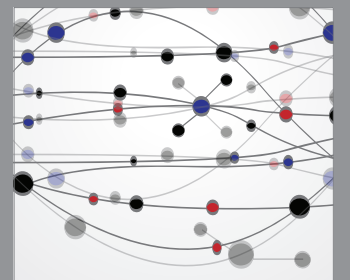

The Scientific World Journal
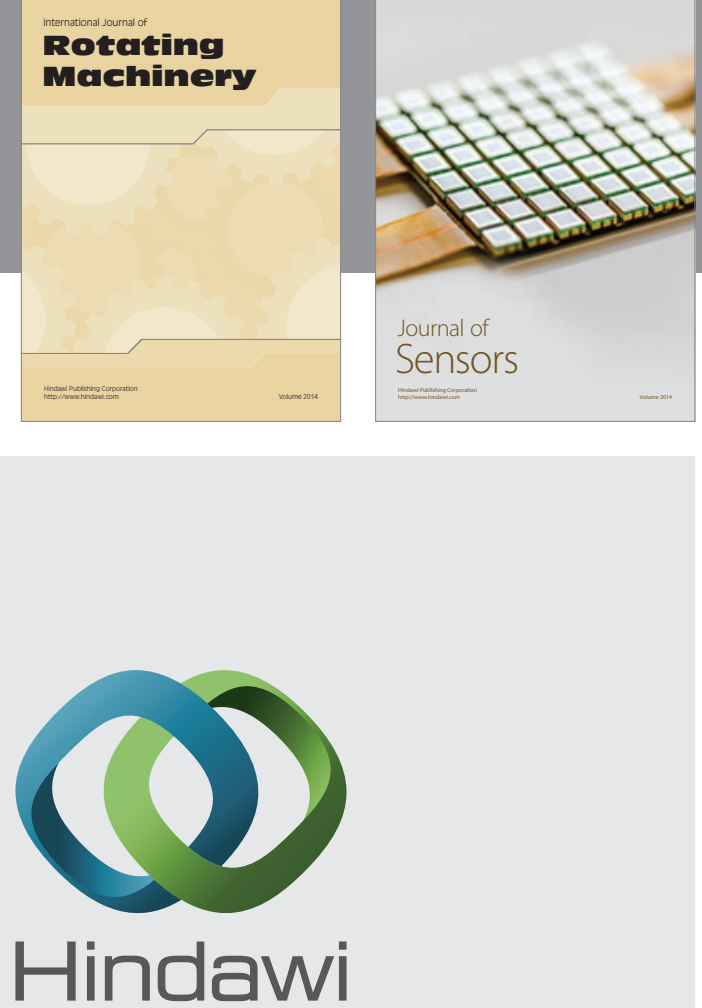

Submit your manuscripts at http://www.hindawi.com
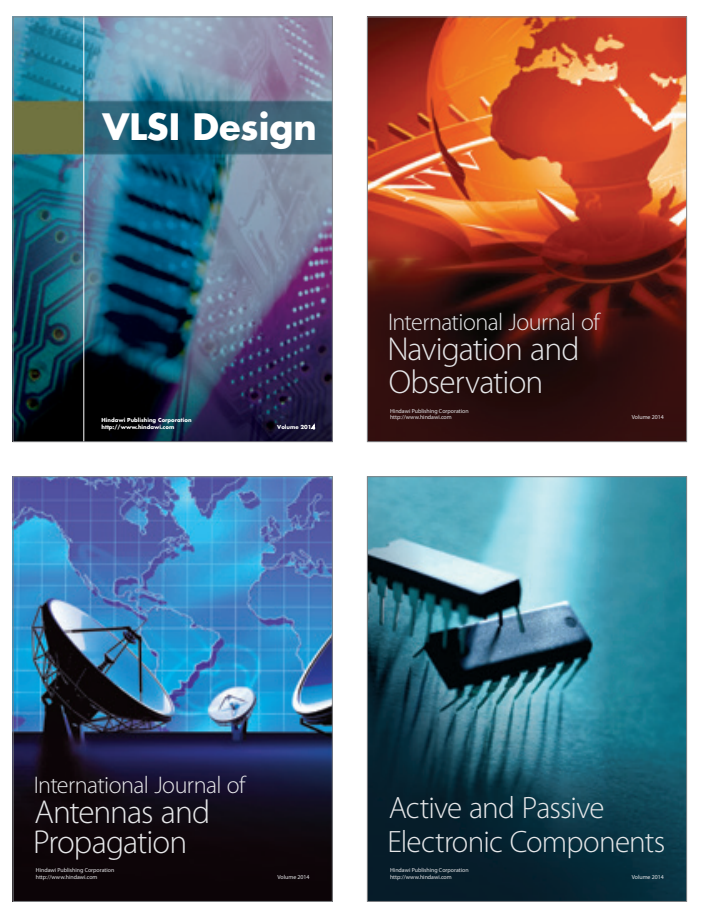
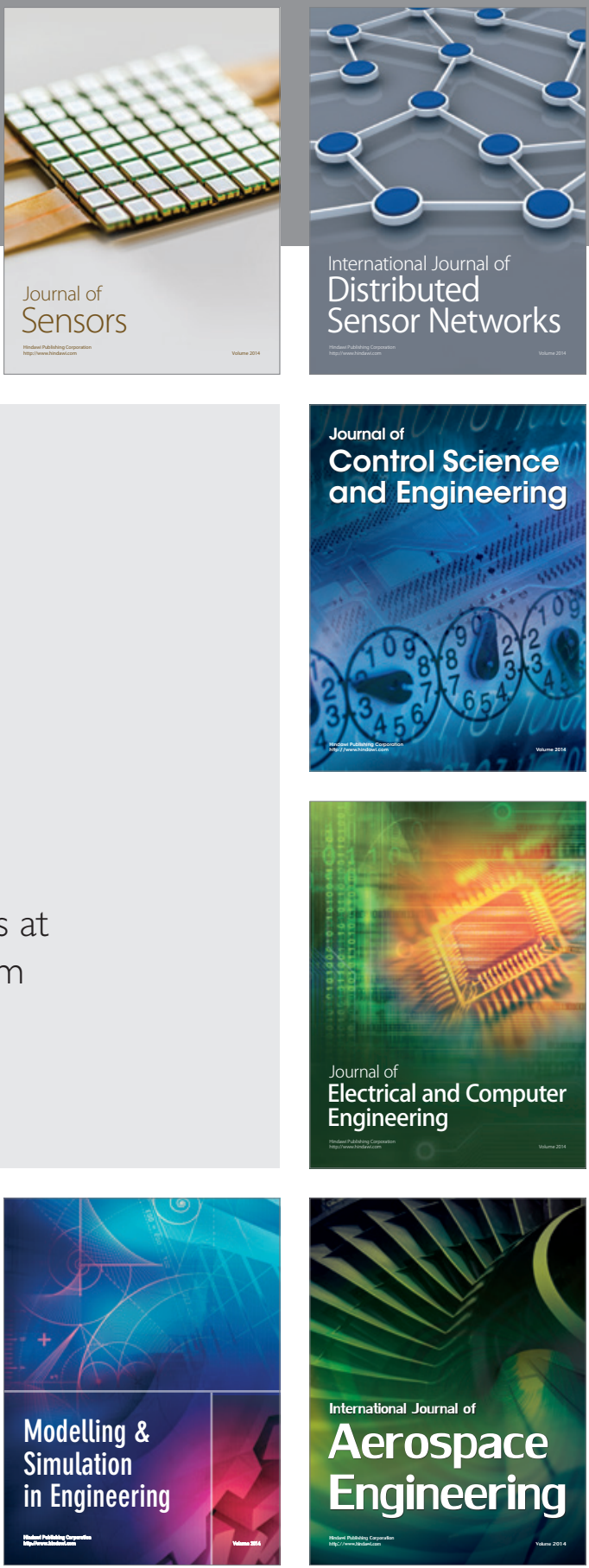

Journal of

Control Science

and Engineering
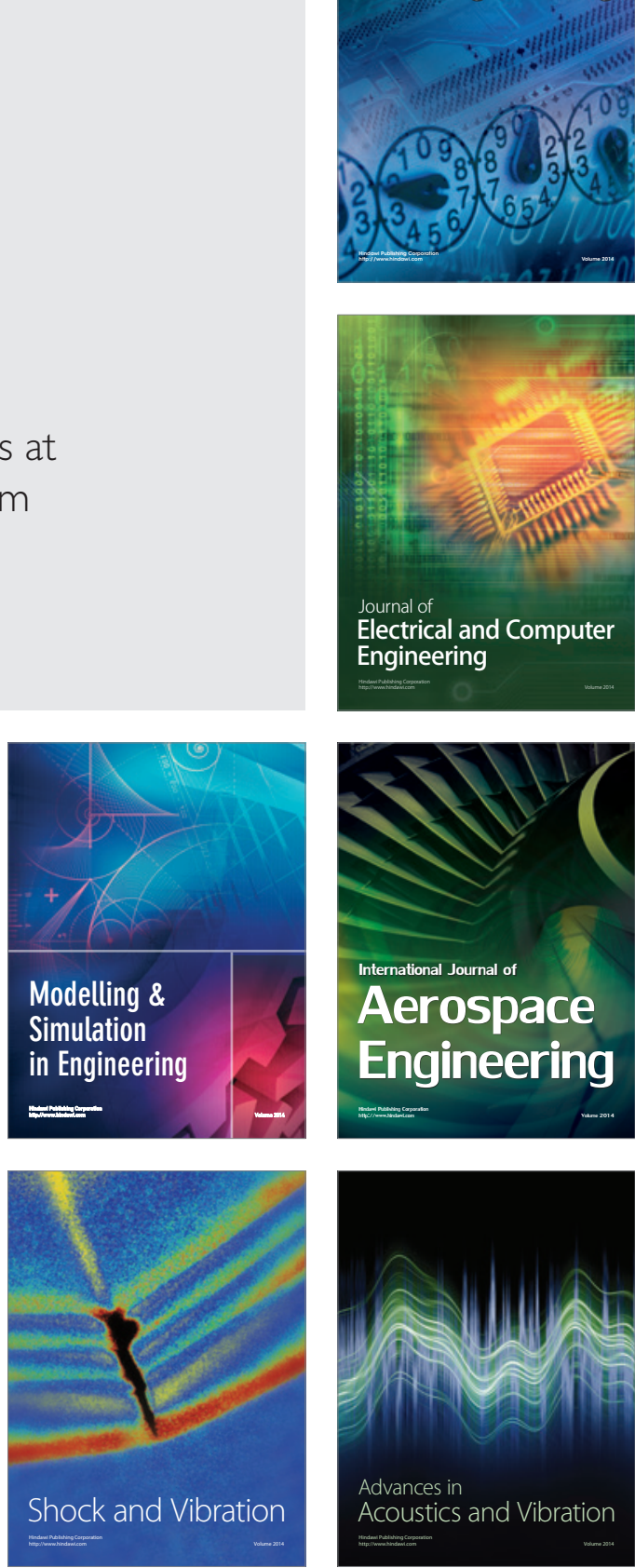